Revue d'histoire de l'Amérique française

ZAS REVUE D.HISTOIRE DE L'AMÉRIQUE FRANÇAISE

\title{
Denonville et ses captifs iroquois (suite et fin)
}

\section{Jean Leclerc}

Volume 15, numéro 1, juin 1961

URI : https://id.erudit.org/iderudit/302094ar

DOI : https://doi.org/10.7202/302094ar

Aller au sommaire du numéro

Éditeur(s)

Institut d'histoire de l'Amérique française

ISSN

0035-2357 (imprimé)

1492-1383 (numérique)

Découvrir la revue

Citer cet article

Leclerc, J. (1961). Denonville et ses captifs iroquois (suite et fin). Revue

d'histoire de l'Amérique française, 15(1), 41-58. https://doi.org/10.7202/302094ar d'utilisation que vous pouvez consulter en ligne.

https://apropos.erudit.org/fr/usagers/politique-dutilisation/ 


\title{
DENONVILLE ET SES CAPTIFS IROQUOIS *
}

\author{
JEAN DE LAMBERVILLE, S.J. \\ ET LES QUARANTE DÉLĖGUÉS IROQUOIS
}

S'il est un document qui jeta de la confusion dans l'histoire de l'expédition de Denonville, c'est bien la lettre que le P. de Lamberville écrivit le 23 janvier 1695 à un ami, missionnaire en Chine. ${ }^{1}$ Cette lettre, Charlevoix l'utilisa mal à propos et les autres historiens par la suite marchèrent sur ses traces sans mettre en doute ses affirmations. Ce document contient certes des inexactitudes et des exagérations évidentes et cependant il fourmille de détails véridiques. C'est qu'il renferme une optique des événements et des personnes qui prend son point d'appui dans la psychologie du P. de Lamberville. Si cette lettre a conduit à des jugements sommaires sur les événements de l'expédition, c'est vraisemblablement qu'on n'a pas suffisamment fait effort pour la comprendre en la replaçant au milieu des faits. La situation du P. de Lamberville présente quelque chose de particulier. Ce Père et son frère Jacques furent les deux derniers missionnaires qui purent résister aux mauvais traitements des Iroquois. ${ }^{2}$ Devant l'opposition tenace de ces Indiens les autres missionnaires jésuites furent obligés de quitter cette mission les uns après les autres. Mais de Lamberville, par sa personnalité, par son esprit de conciliation et de franchise, réussit à tenir le coup jusqu'en 1687. Il fut, par la force des choses, comme un centre sur lequel convergèrent les oppositions entre les Français, les Iroquois et les Anglais, et il sut, par adresse diplomatique comme par un déploiement d'énergie considérable, concilier les différents points de vue ainsi que temporiser avec tous, conservant la paix essentielle à son action missionnaire. Il y était parvenu parce que ses démarches diplomatiques reposaient sur un grand

* Voir première partie de cet article, dans notre Revue, XIV : 545-558.

1 Thwaites, LXIV: 238. Lettre du P. Jean de Lamberville à un Père missionnaire de Chine, Paris, 23 janvier, 1695.

2 Réponse de Den. à Dongan, 22 août 1687. 
amour de la vérité. Il disait à La Barre en 1684 qu'il préférait se retirer plutôt que de tromper les Iroquois $;^{3}$ ce passage est à retenir si l'on veut comprendre sa lettre du 23 janvier 1695. Cet amour de la vérité toutefois ne pouvait pallier à un manque d'information et par suite à un manque de compréhension des événements et des personnes, de Denonville en particulier. Parce qu'il n'admettait pas le procédé dont Denonville se servit pour le faire sortir d'Onontagué, ce qui était pour lui un manque de franchise, l'appréciation qu'il porte de ce gouverneur dans cette lettre en fut faussée. D'autre part, parce qu'il s'était habitué au point de vue iroquois mais qu'il ne saisissait pas toute la portée de leur politique militaire, et que d'autre part il ignorait ou voyait mal les facteurs que Denonville détenait pour justifier son entreprise, il fut enclin à voir en ce gouverneur et dans son entourage des fauteurs de guerre. Avant de se servir de sa lettre du 23 janvier, il importe donc de rétablir les faits à partir des documents circonstanciés et plus près des événements comme sont les narrations de Denonville et de de Baugy. Ensuite on peut y revenir comme c'est le cas pour le recueil de Gédéon de Catalogne. Nous ne prétendons pas ici tout expliquer dans cette lettre, car ce travail serait trop long; nous tenterons seulement de jeter quelque lumière sur certains points qui ont paru obscurs, voire invraisemblables.

De Lamberville a-t-il rassemblé 40 délégués iroquois comme il le prétend dans sa lettre ? Pour répondre à cette question il est nécessaire d'étudier d'abord les circonstances de son départ et de les dater.

I. - Le 29 juin au soir, de Lamberville arrive au camp et rencontre Denonville. ${ }^{4}$ Le lieu de la jonction est situé à 6 lieues $\mathrm{du}$ fort Frontenac soit environ 18 milles (Gannanoque, Ont.). En descendant le St-Laurent, un après-midi lui suffisait pour couvrir cette distance en canot. Le midi, il se trouvait donc au fort. C'était un dimanche, il dut y dire la messe et s'entretenir avec le $\mathrm{P}$. Millet, Dorvilliers et peut-être avec les prisonniers iroquois qu'il connaissait. Il y serait arrivé la veille, le 28. Une

3 De Lamberville à La Barre, 10 juillet 1684.

4 Den., Mém. du Voy.; de Baugy, Journal. 
journée suffisait pour faire la traversée du lac en canot; on peut donc supposer que le 27 au soir, il couchait à l'embouchure de la rivière des Onontagués (Oswego, N.Y.) ou à la Famine (Salmon River, N.Y.) et qu'il était parti d'Onontagué soit le 27 au matin, soit le 26 pendant la journée. Depuis le 24 ou le 25 il était tous les jours attendu au fort Frontenac. ${ }^{5}$

II. - Au moment où quelques Iroquois fugitifs échappés des mains des Français lui apportent la nouvelle de la captivité de leurs frères, il est à 8 lieues d'Onontagué, soit aujourd'hui aux environs de Fulton, N.Y. C'est là que huit des Iroquois les plus notables qui l'accompagnaient, en apprenant la nouvelle, lui dirent de continuer seul sa route, car étant donné qu'il était de bonne foi, ils ne voulaient pas qu'on leur reproche sa mort dans leur nation pour s'être fié à eux. Mais comme ils allaient venger cette perfidie, ils préféraient qu'on le tue avec les Français contre lesquels ils iraient en guerre. De Lamberville, triste et chagriné par ce qui venait d'arriver, poursuivit sa route seul et les huit Notables iroquois s'en retournèrent dans leur pays; ainsi le nombre des 40 députés assemblés par lui en fut pour autant diminué, et conséquemment dans sa lettre du 23 janvier, il ne faudrait pas lire la phrase: "Ils furent au rendez-vous où on les trompa » comme si les 40 députés qu'il assembla étaient inclus dans le pronom «ils ». Comme ces huit députés étaient le reste de la délégation, il faut conclure que les autres étaient partis avant le 26 juin. Ce jour-là l'armée passait le rapide des Galots (Prescott, Ont.).

III. - Le 27 au midi, l'Intendant était à la Galette (Prescott, Ont.) où il rencontrait l'armée. ${ }^{6}$ Son départ du fort Frontenac dut avoir lieu le 26. Il y avait séjourné deux jours, ${ }^{7}$ donc le 24 et le 25. Comme le canot qui, le 25, porta la nouvelle de l'arrêt des Iroquois qui vivaient paisiblement aux alentours du fort à qui on ne fit aucun mal, était parti avant l'arrivée des Iroquois qui pêchaient à l'île d'Otoniato et qu'on avait invités à se rendre au fort pour des présents, ${ }^{8}$ il faut conclure que les premiers

5 De Baugy, Journal, 25 juin.

6 De Baugy, Journal; Den., Mém. du Voy.

7 Champi. au Min., 16 juil. 1687.

8 De Baugy, Journal, 25 juin. 
Iroquois habitant autour du fort furent arrêtés le 24, et ceux de l'île d'Otoniato le 25 ; car au départ de Champigny, vraisemblablement le 26 au midi, tous ces Iroquois avaient été faits prisonniers. Cependant la garnison ne fut renforcie de 100 hommes que le 29 juin. $^{9}$ Entretemps, selon de Catalogne, un Iroquois qui se serait évadé dans des circonstances ingénieuses, serait allé porter la nouvelle à ses compatriotes. ${ }^{10}$ Mais il se pourrait, à cause du stratagème qui l'a frappé que de Catalogne n'ait retenu qu'un nom, alors que d'autres aussi se seraient évadés, car de Lamberville parle de quelques-uns. Il se peut aussi que ceux qui étaient à l'île d'Otoniato ne se soient pas tous rendus au fort, ce que Denonville pressentait quand il fit dire à Ste-Hélène de continuer sa route pour prendre ceux qui traîneraient. ${ }^{11-12}$ Méfiants comme ils étaient, ces Iroquois auraient suivi à distance, puis auraient attendu aux environs du fort pour voir ce qui se passait. Également, le 23 juin, dans la capture effectuée à la Galette par de Boucherville, qui dirigeait un convoi de ravitaillement, il y avait un Goioguen, 4 femmes et un enfant. Où étaient les maris de ces trois femmes? Il semble bien que de Boucherville ne les ait pas saisis et qu'ils aient fui. Ce jour-là Champigny invitait au fort les Iroquois de l'île d'Otoniato, et comme la distance entre cette île et la Galette n'est que de 12 lieues, ce Goioguen pouvait fort bien appartenir à ce groupe d'Iroquois. Il ne semble donc pas que Denonville ait fait un nettoyage complet en montant au fort. Ces fuyards en revenant ont pu se regrouper peu à peu et observer davantage et avec beaucoup de précaution ce qui se passait et lorsque la chose parut certaine que leurs camarades avaient été arrêtés au fort, ils auraient repris la route d'Onontagué. La date du 27, jour de rencontre des fugitifs et du P. de Lamberville, concorde suffisamment avec cette hypothèse. Le fugitif dont parle de Catalogne se serait évadé dans l'après-midi ou le soir du 26 et aurait communiqué la nouvelle aux autres qui attendaient.

\footnotetext{
9 Id., cf. 27 juin. tives.

10 Gédéon De Catalogne, Recueil; Robert Le Blant, Aux sources narra11-12 De Baugy, Journal, 25 juin.
} 
IV. - De Lamberville écrit: «me trouvant encore chez les Iroquois lorsqu'on commença d'arrester leurs députés, les anglois ... informés par des français, qui s'estoient retirés de Kébec pour demeurer parmy eux, des préparatifs qu'on feroit contre les Iroquois ... me donnèrent avis de ce qui se tramait et que l'on se servoit de moy pour tromper les Iroquois... de me retirer en la nouvelle York... Mais ne pouvant me persuader qu'on eust manqué de parole, je me résolus de refuser cette offre et de persuader aux Iroquois de suivre avec moy leurs députés, qu'ils ne scavoient pas encore avoir esté arrestés. »

D'après ce récit l'action se passe peu de temps avant le départ du P. de Lamberville, c'est-à-dire les jours qui précédaient le 26 et au moment où l'on arrêtait leurs députés, soit, semble-t-il, le 24 ou le 25 juin. Cependant il y a ici une simplification des événements. Il est possible que les Anglais aient pu effectivement aller à Onontagué le 24. Les Iroquois, qui rôdaient dans la région de Montréal et qui épiaient le rassemblement des troupes depuis l'arrivée de Denonville et de Champigny, ont pu porter la nouvelle à Dongan et confirmer de cette façon ce que le gouverneur de New-York savait déjà par les déserteurs français. Dongan savait aussi par les Iroquois, le 11 juin, que de Lamberville convoquait une assemblée des députés iroquois. Cette ambassade anglaise est donc plausible, elle a pu se rendre à Onontagué au moment où les premiers Iroquois étaient arrêtés au fort Frontenac, car la marge d'une quinzaine ou d'une vingtaine de jours est, semble-t-il, suffisante pour la transmission de la nouvelle de Montréal à Albany et d'Albany à Onontagué pour la députation anglaise. Cependant il ne faudrait pas admettre pour cette raison que de Lamberville apprenait pour la première fois la marche des troupes. S'il n'a pas voulu se rendre aux arguments des Anglais, c'est que cette nouvelle ne lui avait pas encore été communiquée par la bouche des Français et qu'il ne pouvait pas admettre, sans en avoir la preuve, que Denonville l'avait induit en erreur. Obstiné qu'il était sur le point de la franchise, il a manqué de flair. Il pouvait se demander si Denonville n'avait pas ses raisons d'agir ainsi sans manquer à sa parole. Il ne l'a pas fait. Il se serait rendu compte que son tempérament 
était un obstacle et que la méfiance iroquoise en était un autre. Mais Denonville, qui lui avait pourtant laissé entendre en juin 1686 qu'il maîtriserait un jour les Iroquois, ne pouvait lui donner plus de précisions pour sa propre sécurité. De Lamberville savait donc qu'une expédition se préparait contre les Iroquois mais il ne le savait ni de Denonville ni des Français parce qu'officiellement et pour des raisons de sécurité militaire la convocation de Cataracoui tenait toujours. Ce n'est qu'à la fin de mai que Denonville annonça dans la Colonie son expédition ${ }^{13}$ et il se garda bien d'en avertir les Iroquois. Cependant ceux-ci le savaient déjà. Que s'était-il passé ? Denonville était monté le 22 mai à Montréal pour diligenter les nouvelles qu'il attendait des Pères de Lamberville. Sur les entrefaites Jacques de Lamberville arriva seul d'Onontagué. Il remit au gouverneur les lettres de son frère Jean qui s'était résolu de n'aller à Cataracoui qu'avec les Anciens des Iroquois. ${ }^{14}$ Il apprit vraisemblablement de lui que les Anglais avaient averti les Tsonnontouans d'une attaque prochaine des Français et ils les obligèrent à faire revenir 600 de leurs guerriers partis guerroyer contre les Miamis ainsi qu'un autre groupe qui était allé massacrer les Anglais de Virginie. Il apprit aussi que plusieurs Iroquois avaient fait leur chasse aux environs de Cataracoui. Denonville s'aperçut alors que son secret avait été livré et il craignit beaucoup pour la vie du P. Jean de Lamberville. Qui avait renseigné les Anglais sur les préparatifs de cette expédition ? Gédéon Petit de Chambly s'était enfui en mars du côté de New-York et Salvoye (ou Salvage) de Sorel avait aussi disparu. Dans ces conjonctures Denonville jugea que ces désertions étaient criminelles. ${ }^{15} \mathrm{Le}$ détail qu'en donne de Lamberville n'est donc pas faux, quoiqu'il signifie par Kébec la Colonie, mais l'alerte avait été donnée depuis un mois et demi ou deux mois à ce moment du 24 juin. Jean de Lamberville savait toutes ces nouvelles au milieu de mai, semble-t-il, mais dans sa lettre il a simplifié le récit. Jacques, son frère cadet, ne s'est pas obstiné; il a quitté la mission devant

13 Den. au Min., 8 juin 1687.

14 Id.

$15 I d$. 
le danger et la raison qu'il avait de partir était valable. Par conséquent, Dongan simplifie et exagère lorsqu'il dit à Denonville «when they (les de Lamberville) understood your intentions they thought fit to go without taking leave ». ${ }^{16}$ Le départ de Jacques était bien connu des Iroquois et celui de Jean également. V. - La convocation des 40 délégués iroquois organisée par le P. de Lamberville est-elle plausible ? Par Denonville nous savons que le 8 juin de Lamberville était résolu de n'aller à Cataracoui qu'avec les Anciens des Iroquois. Par de Baugy nous savons que le 23 l'armée apprit la nouvelle que «les considérables délibéraient ». Si nous déduisons environ quatre jours pour la transmission de cette dernière nouvelle, nous pouvons fixer la date de ces délibérations aux environs du 18 et du 19 juin. C'est là que de Lamberville, ne pouvant se rendre à la réalité et pour vaincre la méfiance des Iroquois qui soupçonnaient déjà ce qui se passait, aurait engagé la parole du gouverneur leur disant qu'ils devaient le croire, car étant chrétien il ne pouvait manquer à sa parole. Qui lui avait dit d'agir ainsi ? L'usage que de Lamberville fait du pronom indéfini «on » indique que cette recommandation ne venait pas de Denonville mais de son entourage. A cause du prestige dont jouissait de Lamberville, les délégués se rendirent à ses arguments mais il ne semble pas qu'au fond ils aient été persuadés.

A New-York, Dongan avait déjà reçu la nouvelle de cette convocation le 11 juin et il reprocha ce même jour à Denonville de ne pas l'en avoir averti. ${ }^{17}$ Mais le temps de La Barre était révolu et Denonville fut catégorique dans sa réponse, affirmant sèchement que l'ordre venait bien de lui, puis comme Dongan se montrait mécontent de ce fait, Denonville lui fit remarquer: «C'est me dire que c'est vous qui les avez empêchés d'y venir pour me faire raison de leur violence. $\gg 18$

VI. - Les trente-deux délégués qui seraient partis avant le 26 juin se sont-ils présentés en leur qualité d'ambassadeurs? Les premiers Iroquois arrêtés à Châteauguay, à la Pointe Beaudet et

16 Dongan à Den., 8 sept. 1687.

17 Dongan à Den., 11 juin 1687.

18 Den. à Dongan, 22 août 1687. 
près d'Aulsville, Ont., quoiqu'il y ait eu parmi eux des notables, ne se sont certes pas présentés en qualité d'ambassadeurs, car leur intention y était tout à fait contraire. Ils venaient espionner et faire des prisonniers. De l'aveu de Lamberville lui-même quatre de ces prisonniers étaient des Onontagués, un autre était chef goioguen et leur mission était d'épier la marche de l'armée qu'ils avaient apprise des Anglais, ce qui est significatif pour les autres. ${ }^{19}$ Ils n'avaient donc pas l'intention de venir en ambassadeurs. D'ailleurs ayant été pris le 11 et le 19 juin, ces Iroquois ne durent pas faire partie de la convocation du P. de Lamberville. Le Goioguen arrêté par de Boucherville le 23 ne semble pas non plus s'être présenté en qualité d'ambassadeur. Bien qu'il fût un considérable parmi les Goioguens, il venait pour observer la marche de l'armée. ${ }^{20}$ Ceux qui furent arrêtés le 24 aux alentours du fort étaient des Iroquois qui étaient établis depuis longtemps dans ce lieu et à qui on ne fit pas de mal. Il faut exclure ceux qui furent arrêtés à Gannéious, car lorsqu'ils parvinrent au fort, le $P$. de Lamberville y était depuis quatre jours et le 27 juin, tous ceux qui faisaient partie de son ambassade s'y étaient rendus suivant son récit. Mais où étaient-ils allés ? Il ne reste qu'une possibilité, celle des Iroquois qui pêchaient à l'île d'Otoniato. S'il $\mathrm{y}$ avait de ses ambassadeurs, ils étaient là. Comme ils furent invités au fort le 23 , il y a possibilité qu'ils soient partis d'Onontagué après les délibérations, soit le 19 , le 20 ou le 21 . Il y avait là le fils et le frère de la Grande Gueule. ${ }^{21}$ Il y avait probablement Otasseté, capitaine Oneiout. ${ }^{22}$ Il y avait là aussi des chasseurs. ${ }^{23}$ Quel était leur nombre ? Lorsque Champigny donna le chiffre de ceux qui furent arrêtés aux alentours du fort et de ceux de l'île d'Otoniato, il dit qu'ils étaient 30 hommes. ${ }^{24}$ Il faudrait donc conclure que ceux qui furent arrêtés venant de l'île d'Otoniato ne dépassaient guère 15 ou 20 tout au plus. Et comme

19 De Baugy, Journal, 29 juin.

20 Den., Mém. du Voy., 23 juin.

21 De Baugy, Journal, 25 juin et 1 juill.; Den., Mém. du Voy., 30 juin. 22 Pierre Millet, Onneiout, 6 juil. 1691, Thwaites, LXIV : 66.

23 Den. au Min., 8 juin 1687. Les Iroquois ont chassé aux alentours de Cataracoui; W. J. Eccles, «Denonville et les galériens iroquois 》, RHAF, XIV: (déc. 1960), 420.

24 Den., Mém. du Voy., 27 juin. 
il y avait parmi eux des chasseurs, le nombre des ambassadeurs s'y trouvait très réduit et rien n'indique, d'après les récits de Denonville et de de Baugy, qu'ils se soient présentés en leur qualité d'ambassadeurs, si ce n'est le fils et le frère de la Grande Gueule que Denonville relâcha en leur disant qu'il ne manquait pas à sa parole. ${ }^{25}$ L'île d'Otoniato était d'ailleurs un singulier chemin pour aller au rendez-vous du fort Frontenac, ce qui laisse voir qu'ils n'avaient guère d'intention sérieuse de parlementer et que les paroles de Lamberville ne les avaient guère persuadés. Méfiants qu'ils étaient, ils préféraient attendre et observer, ce qui confirmait les appréhensions de Denonville.

VII. - Même s'il y avait quelques députés, Denonville avait-il ses raisons pour les capturer? Dans toutes les délibérations qu'il avait eues avec eux, les députés iroquois s'étaient toujours informés en premier lieu du nombre d'hommes de l'escorte du gouverneur et ils s'étaient toujours ingéniés pour se trouver en plus grand nombre afin de l'intimider et de se moquer de lui. ${ }^{26}$ On sait que La Barre, dans ses pourparlers de paix à la Famine en septembre 1684, avait été joué d'une façon honteuse par la Grande Gueule (Hotreouati). ${ }^{27}$ Il est donc à se demander si les Iroquois eux-mêmes respectaient ce que les Européens appelaient le droit des gens ? Cette fois, Denonville était décidé qu'on ne se moquerait pas de lui, et qu'il délibérerait les armes à la main. ${ }^{28}$ Comme il ne se fiait plus à leurs paroles et qu'il les savait hostiles, il considéra comme des ennemis ces quelques députés qui ne se présentèrent guère en qualité d'ambassadeurs, faisant partie d'une ambassade assez dispersée et guère représentative puisque plusieurs chefs avaient déjà été arrêtés pour espionnage.

VIII. - De Lamberville dit aussi qu'il obtint le renvoi de sept ou huit personnes pour les services qu'elles lui avaient rendus à l'occasion. Ce fait est plausible. Car si de Baugy affirme qu'une personne fut libérée à savoir le fils de la Grande Gueule, Denonville par contre dit qu'il en renvoya deux: le fils et le frère de la

25 De Baugy, Journal, 1 juil.

26 Den. au Min., 8 juin 1687.

27 De Meulles au Min., 10 oct. 1684.

28 Den. au Min., 8 juin 1687. 
Grande Gueule, ce qui n'exclut pas qu'il y ait eu parmi eux des personnages moins importants, ainsi que des femmes et des enfants. Mais il garda des Onontagués avec l'intention de s'en servir, car il voulait en arriver à des accommodements de paix. De Callières affirme que Denonville sépara les Onontagués des autres et les renvoya à leurs nations pour qu'ils persuadent les autres Iroquois de venir négocier la paix. ${ }^{29}$ Quand eut lieu ce renvoi ? À la mi-octobre, Denonville envoie 4 Iroquois venant d'Oneiout et Onontagué afin qu'ils essaient de persuader leurs compatriotes de se dissocier des Tsonnontouans. ${ }^{30}$ À la fin de mai 1688, les Onontagués, les Goioguens et les Oneiouts descendirent à Montréal pour négocier la paix. ${ }^{31}$ Quand de Lamberville dit qu' « on a alors tenté en vain de faire la paix avec les Iroquois », nous ne croyons pas que le Père signifie par là que ces tentatives eurent lieu après la capture des prisonniers et avant l'attaque de Denonville contre les Tsonnontouans. C'est sa composition qui est en défaut, son style qui est décousu.

IX. - Est-il plausible que de Lamberville ait rencontré Denonville en 1686 ? Il ne semble pas y avoir de doute à ce sujet. Il était à Montréal au début de juin. ${ }^{32}$ Si de Lamberville dit qu'il l'a rencontré à Kébec, nous croyons comme dans le cas de Gédéon Petit de Chambly qu'il signifie dans la Colonie. Denonville à ce moment préparait en secret la synchronisation des mouvements de son expédition de 1687. Le 6 juin, il écrivait à La Durantaye pour lui donner l'ordre d'intercepter les canots anglais à Détroit et au portage de Toronto et de se tenir prêt pour le printemps suivant. ${ }^{33}$ À du Lhut, il demandait d'aller à Détroit avec 50 hommes et lui enjoignait de recevoir ce que le P. Enjalran, S.J., lui communiquerait de sa part. ${ }^{34}$ A La Forest, commandant au fort St-Louis des Illinois, il ordonne sans autre ordre ultérieur d'aller

29 A Seignelay, Mém. du Ch. de Callières, gouverneur de Montréal, sur l'Etat des Affaires. Ce mémoire est sans date aux Archives de Québec. D'après le contexte il semble qu'il ait été écrit par de Callières au début de 1689 pendant son séjour à Versailles.

30 Den., Mém. Etat des Aff., 27 oct. 1687. juin 1688 .

31 Montréal, Déclaration des Iroquois devant M. de Denonville, 15

32 Den. au Min., 12 juin 1686.

33 Ville-Marie, Den. à La Durantaye, 6 juin 1686 .

34 Ville-Marie, Den. à du Lhut, 6 juin 1686. 
le printemps suivant avec de Tonty à la pointe du lac Erié. ${ }^{35}$ Le P. Enjalran était aussi à Montréal; il devait apporter ces lettres en retournant à Missilimakinac, mais en passant par le fort Frontenac où les Iroquois devaient lui remettre des prisonniers. $^{36}$ Une affaire épineuse s'était développée. Scoutache, un Huron, s'était livré aux Iroquois avec 70 de ses compatriotes plus 36 Outaouais petits et grands. De Lamberville réussit à convaincre les Iroquois de remettre ces transfuges, et fut appelé à Montréal par Denonville pour régler avec les Iroquois les détails de cette affaire. ${ }^{37}$ C'est à ce voyage vraisemblablement qu'il réfère dans sa lettre lorsqu'il dit que le gouverneur le fit venir à Kébec, mais l'affaire Scoutache ne fut qu'une diversion; ce que Denonville voulait de lui, c'était de synchroniser sa convocation des Iroquois à Cataracoui avec le mouvement des troupes et garder ainsi son expédition secrète. Mais Denonville, tout en lui laissant entendre qu'il viendrait à bout des Iroquois, ne lui livra pas son secret. Cependant de Lamberville, à son retour à Onontagué, voulant toujours être franc avec les Iroquois, leur livra indiscrètement ce qu'il croyait être son secret, à savoir que les Français viendraient les attaquer quand les maïs seraient presque mûrs. ${ }^{38}$ Mais, pour avoir voulu trop prendre la part des Iroquois, il se trompait en interprétant ainsi les paroles de Denonville.

X. - De Lamberville parle de deux perfidies qui se seraient succédé rapidement. Nous voyons là les deux temps de l'arrêt des Iroquois au fort Frontenac. Le premier serait celui du 24 juin, lorsque l'on arrêta les Iroquois qui vivaient paisiblement autour $\mathrm{du}$ fort; le second serait celui du 25, jour où l'on captura ceux qui vinrent de l'île d'Otoniato. Le P. de Lamberville dit en effet que dans cette deuxième perfidie le $\mathrm{P}$. Millet eut beaucoup à souffrir. Nous savons que Bayard affirme que c'est le P. Millet qui invita les Tsonnontouans et que ce sont les Français qui les

35 Ville-Marie, Den. à La Forest, 6 juin 1686.

36 Den. au Min., 12 juin 1686.

$37 \mathrm{Id}$.

38 W. J. Eccles, «Denonville et les galériens iroquois 》, RHAF, XIV : (déc. 1960 ), 422, note 45. The Livingston Indian Records, 1663-1723, 103, Proposal of the 3 Maquase Sachems (Juil. 1686). 
mirent dans les fers. ${ }^{39}$ Il semble bien d'après ces récits que les souffrances du P. Millet furent avant tout morales, c'est-à-dire qu'il souffrit beaucoup de ce qu'on lui fit inviter les Iroquois pour un motif et qu'on les captura pour un autre. A quels Iroquois s'était-il adressé ? Les faits de sa captivité sont significatifs. En 1689, l'armée iroquoise qui descendait à Montréal pour effectuer le massacre de Lachine passa non loin du fort Frontenac. Valrenne, commandant du fort, l'aperçut et envoya porter la nouvelle à Montréal. Cependant quelques Iroquois s'arrêtèrent au fort et demandèrent le chirurgien, et le $P$. Millet, sous prétexte qu'il y avait un malade qui avait besoin d'être saigné. Lorsqu'ils s'éloignèrent du fort, ils le firent prisonnier, le frappèrent, l'insultèrent, lui dirent que les Français étaient des perfides, ${ }^{40}$ et, comme il avait déjà été jugé par les Iroquois et les Anglais, ils l'amenèrent jusqu'à l'île d'Otoniato où ils se préparèrent à le brûler à petit feu. Puis, ils se ravisèrent et l'envoyèrent sous escorte à Oneiout où à leur retour en octobre ils se concertèrent et décidèrent, après bien des hésitations, de le naturaliser iroquois en remplacement d'Otasseté, capitaine oneiout qui était mort en France. ${ }^{41}$ Il y exerça ainsi son ministère pendant cinq ans. Ce fait semblerait donc une indication que la deuxième perfidie dont parle de Lamberville se rapporte à la capture des Iroquois de l'île d'Otoniato invités au fort par Champigny.

XI. - De Lamberville affirme qu'il a reçu des lettres qui lui enjoignirent «de se retirer d'Onontagué par quelques moyens parce que les Français montaient en guerre contre les Iroquois », mais que ces lettres arrivèrent trop tard. Peut-on admettre cette assertion ? Denonville affirme à l'arrivée de Lamberville, le 29, qu'il l'a «envoyé quérir sous prétexte d'amener les considérables des Iroquois pour négocier avec eux sur les manières de pacifier nos différends ». Comme le 8 juin, en écrivant au ministre, Denonville dit que « notre peine à présent est de savoir si le pauvre $\mathrm{P}$. de Lamberville, missionnaire jésuite, qui est encore à

\footnotetext{
39 London Archives, Docu. on Colonial History. Account of the proceedings of the 5 Nations against the French in Canada. Extract of Bayond's letter, sept. 23, 1689, Signed N. Bayard.

40 De Lamberville, Paris, 23 jan. 1695, Thwaites, LXIV: 238 sq.

41 Pierre Millet, Onneiout, 6 juil. 1691, Thwaites, LXIV: 66 sq.
} 
Onontagué se retirera de leurs mains sur le grand bruit de guerre que l'on n'a pas pu empêcher de se rependre sur les préparatifs extraordinaires qu'il a fallu faire, quoique j'ay toujours publié que je n'allais qu'à l'assemblée générale projetée à Cataracouy où je ne voulais pas être insulté ni moqué » et un peu plus haut: " Tout cela me fait craindre que le pauvre père n'ait de la peine à se retirer des mains de ces barbares ce qui m'inquiète fort », et que d'autre part il ne dit pas au ministre qu'il l'a envoyé chercher, il faudrait conclure que cette décision fut prise après le 8 juin. Le moment du départ du courrier chargé de cette mission pourrait être fixé, soit avec celui des premiers éclaireurs, soit au plus tard avec Champigny qui montait avec une partie du convoi. Champigny mit 10 jours à Monter; il en fallait trois de plus pour se rendre à Onontagué, ce qui fixerait la réception des lettres au plus tard le 26 et au plus tôt le 23. Comme Denonville l'a envoyé chercher officiellement pour négocier, d'autres ont pu lui écrire ces lettres, et en particulier son frère qui venait d'apprendre en arrivant à Montréal ce qui se passait. Ses supérieurs également ou quelques officiers ont pu tout aussi bien le presser de sortir d'Onontagué. Ces lettres ont pu être remises au même courrier. Cependant de Lamberville dit qu'il les a reçues trop tard; ce devait être au moment où il était en train de partir, après qu'il eut refusé l'offre des ambassadeurs anglais de se retirer à NewYork, ne voulant pas croire de leur bouche qu'on l'avait trompé, et après que la plupart des députés se fussent mis en route, ce devait être au soir du 25 ou dans la journée du 26. De plus comme le courrier l'invitait officiellement au nom de Denonville et que d'autre part les lettres le faisaient hésiter et qu'il ne savait pas que déjà on arrêtait les Iroquois, il se devait d'attendre de plus amples renseignements avant de livrer aux Iroquois le contenu des lettres. Il se peut aussi qu'il crut comme au temps de La Barre qu'il était en mesure d'arranger les choses, aussi n'aurait-il pas livré avant l'heure ces nouvelles qui lui paraissaient contraires aux ordres reçus. Son assertion semble donc plausible.

XII. - De Lamberville affirme qu'il trouva au fort 200 prisonniers. Son assertion est exacte, non toutefois le 29 juin, jour de son arrivée, mais le 3 juillet. Au sujet des 13 Iroquois dont il 
déclare qu'ils furent les seuls à revenir de France, son affirmation est exacte. ${ }^{42}$ Quand il dit que les autres sont morts de misère, on peut en douter; mais aussi on peut comprendre son optique. Il parle en européen, il n'a probablement jamais vu ce qui se passait aux galères et il ignorait les recommandations faites par Denonville et le ministre à leur sujet. Il parlait en somme comme les gens de son temps qui s'imaginaient que là résidait le pire des châtiments. Qu'il en soit mort en France, il n'y a rien d'étonnant à ce fait lorsque l'on sait à quel point tous ces Indiens d'Amérique étaient vulnérables aux microbes des Européens. Les huit cents soldats arrivés à la fin de mai avaient apporté la rougeole. Dixsept d'entre eux étaient morts pendant la traversée, cent trente avaient été hospitalisés à l'Hôtel-Dieu de Québec.43 La contagion s'était rapidement répandue dans la Colonie et elle avait fait de tels ravages qu'en novembre on pouvait compter 500 habitants et 300 Sauvages qui furent emportés dans cette épidémie. ${ }^{44}$ Sur le Fourgon qui transporta ces captifs iroquois, on comptait en juillet 17 hommes d'équipage malades à l'hôpital, ${ }^{45}$ ce qui fit retarder le départ de ce navire. Les prisonniers iroquois s'embarquaient donc sur un navire contaminé et laissaient derrière eux une population également contaminée. Ce n'est certes pas le fait d'avoir marché nu-pieds qui peut être qualifié de mauvais traitements, ce qui pourtant désola Denonville, ${ }^{46}$ car ces Sauvages y étaient habitués. Lorsqu'ils furent rappelés à Rochefort, il n'en restait plus que vingt-et-un ${ }^{47}$ et pour les consoler quelque peu, on leur donna Lemoyne de Sérigny, fils de Charles Lemoyne de Montréal, pour les assister. Il était passé en France en 1686 pour devenir garde de la marine. ${ }^{48}$ Il savait l'iroquois comme ses frères Ste-Hélène, d'Iberville, Maricourt.

42 W. J. Eccles, «Denonville et les Galériens iroquois 》, RHAF, XIV : (déc. 1960 ), 425, note 53.

43 Champi. au Min., 16 juil. 1687.

44 Den. et Champi. au Min., 6 nov. 1687.

45 Champi. au Min., 16 juil. 1687.

46 Den. à Seignelay, Mém. Etat des Aff., 10 août 1688.

47 Le rôle de ces captifs se retrouve dans Coll. des Manuscrits, vol. I, J. Blanchet (Québec, 1883) : 454.

48 Champi. au Min., 16 nov. 1686, Dugué de Ste Thérèse passa aussi avec Sérigny. 
XIII. - De Lamberville affirme aussi que Denonville alla attaquer les Iroquois par un autre chemin. Il est clair ici qu'il n'a pas su et n'a pas compris la stratégie de Denonville. En partant de Montréal, Denonville avait arrêté un plan précis. Pour permettre à de Tonty, Du Lhut, La Durantaye et aux alliés d'arriver à Niagara sans être inquiétés par les Tsonnontouans, Denonville avait décidé qu'il arriverait sur le lac Ontario le premier afin que les Iroquois viennent d'abord le chercher, ensuite qu'il ferait un mouvement de diversion en passant par le sud du lac pour que les Iroquois ne sachent pas sur quelles nations: Oneiout, Onontagué, Goioguen ou Tsonnontouan il ferait porter son attaque. ${ }^{49}$ L'affirmation de Lamberville n'est donc pas fausse, mais elle a besoin d'être expliquée, car il semble bien qu'il ait toujours cru que Denonville voulait répéter le mouvement de La Barre et aller à la Famine puis chez les Oneiouts et les Onontagués, ce qui indique aussi que le secret de Denonville fut bien gardé. On lui a peut-être laissé entendre que c'était parce qu'il avait amené des députés que l'on n'avait pas attaqué les Onontagués, mais la véritable raison était bien plutôt que l'organisation, le nombre d'hommes et les fatigues d'une semblable expédition ne permettaient pas à Denonville d'attaquer successivement et en un seul été toutes les nations iroquoises et qu'il devait pour une première année concentrer ses forces sur une seule nation; celle qui fut choisie en l'occurence fut celle des Tsonnontouans, nation plus arrogante et plus forte. Après sa campagne Denonville dit qu'il aurait bien aimé aller chez les autres nations, mais la grande fatigue de ses troupes ne le lui permit pas. ${ }^{50}$ A cause des limites de ses moyens militaires, Denonville usa de diplomatie et essaya de dissocier les autres nations des Tsonnontouans; voilà pourquoi il fit valoir auprès des Onontagués ses volontés de paix et qu'il se servit du P. de Lamberville à cet effet. ${ }^{.1}$ Mais tous ces Iroquois étaient des ennemis de la Colonie et faisaient la guerre aux alliés. Denonville, au retour de sa campagne, projeta d'attaquer simultanément l'année suivante toutes les nations iroquoises en envoyant une

49 Den. au Min., 8 juin 1687.

50 Den. au Min., 25 août 1687.

51 Champi. et Den. au Min., 6 nov. 1687. 
armée du côté du lac Champlain et une autre du côté du lac Ontario. ${ }^{52}$ La cour lui envoya même un plan de campagne détaillé dans ce dessein afin de réduire les Iroquois en deux ans. ${ }^{53}$ Mais les mortalités dans la Colonie à l'automne de 1687, celles qui eurent lieu à l'hiver et au printemps de 1688 au fort Frontenac et au fort Niagara, l'empêchèrent d'exécuter ses projets. Il chercha alors à négocier et c'est pour cette raison que les pourparlers de Montréal eurent lieu en juin 1688.

XIV. - Y avait-il des Agniers parmi les prisonniers capturés par Denonville ? Deux faits sembleraient indiquer qu'il y en ait eu. Lorsque Mlle d'Alonne fut capturée au fort Frontenac à la fin d'octobre ou au début de novembre avec trois soldats de la garnison, Dorvilliers envoya le P. de Lamberville pour parlementer près du fort avec les Oneiouts, les Onontagués et les Agniers qui avaient fait la capture. Comme le P. de Lamberville leur demandait pourquoi ils faisaient des prisonniers français puisqu'ils n'étaient pas en guerre contre eux mais contre les Tsonnontouans, ils rétorquèrent à leur tour demandant pourquoi les Français avaient fait prendre tant de leurs gens. ${ }^{54}$

En 1688, les Agniers et les Loups furent les plus acharnés à faire du dégât dans la Colonie. Ils firent éruption en juillet par la rivière St-François, ravagèrent partout sur leur passage, principalement à Sorel, Contrecœur et St-Ours. L'envoyé de Dongan les ramena à son retour de Montréal en août. Mais on avait appris de quelques-uns d'entre eux que Dongan leur avait dit «que le vray moyen de retirer leurs prisonniers, c'étoit d'en faire sur nous $\gg .^{55}$

XV. - En 1687, Jean de Lamberville avait 53 ans et son frère cadet Jacques en avait 46. En 1695, au moment où il écrit à son

52 Den. à Seignelay, Mém. sur la nécessité de faire la guerre, nov. 1686. Den. au Min., 25 août 1687; 7 nov. 1687, Den. au Min., Etat des Aff., 27 oct. 1687.

53 Versailles, 8 mars 1688, Mém. sur la guerre à faire aux Iroquois. 54 Québec, 30 oct. 1688, Relations des événements de la guerre et dispositions des affaires du Canada (le nom de l'auteur n'est pas indiqué, nous savons seulement qu'il était dans la Colonie depuis 23 ans).

55 Id.; Den. au Min., 25 août 1687 . Denonville affirme que parmi les captifs qu'il a fait quelques-uns sont proches parents de nos Sauvages chrétiens. Or les Sauvages Chrétiens du Sault et de la Montagne étaient pour beaucoup des Agniers, ce qui indiquerait aussi que parmi les prisonniers il y avait des Agniers. 
ami missionnaire en Chine, Jean a 60 ans; il est à Paris depuis trois ans et occupe la charge de procureur de la mission «en attendant l'heureux moment qui me fasse repasser la mer pour aller finir le peu de jours qui me restent dans notre cher Canada ». Mais à cet âge ses chances de retourner dans sa mission étaient considérablement réduites. Il ne semble pas toutefois que la maladie du scorbut qu'il contracta au fort Frontenac en février 1688 et dont il mit deux ans et demi à se remettre, ainsi que les privations et les mauvais traitements qu'il endura chez les Onontagués, aient pour autant diminué ses chances d'une longue vie. Il mourut à Paris en 1714 à l'âge de 80 ans. ${ }^{56}$ Son frère Jacques l'avait déjà précédé dans la tombe. Il était mort au Sault StLouis en 1711 à l'âge de 70 ans..$^{57}$

\section{CONCLUSION}

Si Jean de Lamberville a gardé un souvenir amer de cette guerre, dans laquelle il a vu se briser ses espérances apostoliques malgré ses efforts de franchise et d'accommodement, il n'a pas perdu pour autant tout le mérite qu'il s'est acquis dans ses démarches de conciliateur. Le rôle qu'il a joué chez les Iroquois pendant cette période pour empêcher la guerre est un rôle de premier plan. Et les lettres qu'il a écrites pendant son séjour à Onontagué sont généralement objectives et partant très utiles à l'historien. Si cependant sa lettre du 23 janvier 1695 paraît étrange sur plusieurs points, c'est qu'elle nécessite un effort de compréhension particulier qui ne peut être obtenu que par sa remise en place dans les événements de ce temps, et ceux-ci ne peuvent être reconstitués sûrement qu'avec les autres sources historiques. En conséquence, lorsque de Lamberville dit qu'il assembla 40 délégués, qu'ils allèrent au rendez-vous où on les trompa et les mit dans les fers, qu'on les transporta en France où ils périrent de misère, il ne faut pas oublier qu'il voit le passé au moyen d'une optique déficiente, qu'il simplifie les événements, qu'il généralise sans entrer dans les détails, car il ne s'agissait pour lui somme toute que d'une lettre sans conséquence qu'il adressait à son con-

56 Nécrologe de la Cie de Jésus, Prov. du Bas-Canada, le 6 fév. 57 Ibid., le 18 avril. 
frère de Chine, et dans laquelle il était superflu d'entrer dans tous les détails. Il se permit donc certaines exagérations qu'il ne se serait certes pas permises, s'il avait écrit à Denonville luimême, au ministre ou au roi. On voit mal qu'il ait utilisé l'ironie pour décrire les talents militaires de Denonville comme il l'a fait dans l'intimité de sa lettre, s'il avait écrit officiellement, par exemple, à de Callières ou au ministre, ce qui aurait paru tout de suite une fausseté, car Denonville avait d'authentiques qualités militaires. Mais à partir de l'été de 1688, on s'était habitué dans la Colonie à rejeter tous les malheurs de la guerre sur ce gouverneur, et celui-ci devant la complexité des affaires demanda avec une probité et une humilité qui n'est pas sans nous émouvoir d'être remplacé «par un plus habile homme que moi ».58 Cependant si Denonville n'a pu conduire cette guerre à bonne fin comme il le prévoyait au printemps de 1686, on ne saurait justement l'en blâmer, car la mortalité due à la maladie faucha dans les rangs de ses troupes et dans ceux des habitants; le roi ne lui envoya pas tous les secours qu'il requérait à cette fin: en 1688 il ne lui envoya que 300 soldats ${ }^{59}$ et en 1689 il refusa d'en envoyer; ${ }^{60}$ il le mortifia par la restriction des crédits; ${ }^{61}$ enfin l'intervention de Dongan à partir du 6 août 1687 annula les avantages que lui avait donnés son expédition. Autant de facteurs que de Lamberville semblait encore ignorer en 1695.

Il demeure donc que cette lettre du 23 janvier ne peut être utilisée que sous réserve et après un contrôle qui ne peut venir que des autres sources de documentation.

JEAN LECLERC, S.J.

\section{Collège des Jésuites, Québec.}

58 Den. à Seignelay, 10 août 1688; Mém., Etat des Aff., «Les affaires de ce pays ont été si outrées que les remèdes pour les rétablir dans leur première réputation passent ma portée et ne demandent pas moins que la grandeur du Roi et un plus habile homme que moi pour les rétablir. »

59 Mém. du Roy aux Sieurs de Den. et Champi., 8 mars 1688.

60 Ordre du Roi, 1689,30 avril. Le roi donne l'ordre de réduire à 28 les 35 compagnies, dont la diminution était de 332 soldats. Il trouve la diminution considérable et refuse d'envoyer de nouveaux soldats.

61 Den., Mém. Etat des Aff., 27 oct. 1687, « La dépense que vous faites me mortifie plus que vous ne sauriez croire et d'autant plus que vous désirez la (guerre) voir finir. Je voudrais vous servir à votre gré mais cela ne se peut en ce rencontre ». Den. et Champi. au Min., 6 nov. 1687; Den. au Min., 7 nov. 1687; Mém. du Roy aux Sieurs de Den. et Champi., 8 mars 1688. 